\title{
Prevalence of Lipohypertrophy and Associated Risk Factors in Insulin- Treated Patients With Type 2 Diabetes Mellitus
}

\author{
Mo'men Al Ajlouni ${ }^{1}$; Mousa Abujbara ${ }^{2}$; Anwar Batieha ${ }^{3}$; Kamel Ajlouni ${ }^{2,{ }^{*}}$ \\ ${ }^{1}$ Prince Sultan Military Medical, Riyadh, Saudi Arabia \\ ${ }^{2}$ The National Center for Diabetes, Endocrinology and Genetics, Amman, Jordan

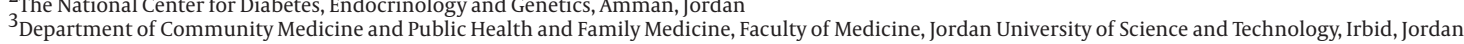 \\ *Corresponding author: Kamel Ajlouni, The National Center for Diabetes, Endocrinology and Genetics, P. O. Box: 13165, Amman, Jordan. Tel: +96-265347810, Fax: +96-265356670, \\ E-mail: ajlouni@ju.edu.jo
}

Received: July 1, 2014; Revised: September 27, 2014; Accepted: December 2, 2014

\begin{abstract}
Background: Secondary failure of oral hypoglycemic agents is common in patients with type 2 diabetes mellitus (T2DM); thus, patients often need insulin therapy. The most common complication of insulin treatment is lipohypertrophy (LH).

Objectives:This study was conducted to estimate the prevalence of LHamong insulin-treated patients with Patients with T2DM, to identify the risk factors for the development of $\mathrm{LH}$, and to examine the association between $\mathrm{LH}$ and glycemic control.

Patients and Methods: A total of 1090 patients with T2DM aged 20 to 89 years, who attended the diabetes clinics at the National Center for Diabetes, Endocrinology, and Genetics (NCDEG, Amman, Jordan) between October 2011 and January 2012, were enrolled. The presence of $\mathrm{LH}$ was examined by inspection and palpation of insulin injection sites at the time of the visit as relevant clinical and laboratory data were obtained. The LH was defined as a local tumor-like swelling of subcutaneous fatty tissue at the site of repeated insulin injections. Results: The overall prevalence of LH was $37.3 \%$ (27.4\% grade $1,9.7 \%$ grade 2 , and $0.2 \%$ grade 3 ). The $\mathrm{LH}$ was significantly associated with the duration of diabetes, needle length, duration of insulin therapy, lack of systematic rotation of insulin injection sites, and poor glycemic control.

Conclusions: The LH is a common problem in insulin-treated Jordanian patients with T2DM. More efforts are needed to educate patients and health workers on simple interventions such as using shorter needles and frequent rotation of the insulin injection sites to avoid LH and improve glycemic control.
\end{abstract}

Keywords: Lipodystrophy; Insulin; Diabetes mellitus

\section{Introduction}

Many patients with type 2 diabetes mellitus (T2DM) need treatment with exogenous insulin at some point during their lifetime because of the secondary failure of oral hypoglycemic medications (1). The United Kingdom Prospective Diabetes Study (UKPDS) showed that 38\% of patients with T2DM need insulin treatment within ten years of diagnosis (1). Insulin therapy is associated with skin-related complications, such as lipoatrophy, lipohypertrophy (LH), edema, and allergy (2). The most common skin-related adverse effect of insulin treatment is LH, which is a local tumor-like swelling of fatty tissue around the subcutaneous insulin injection sites (3-5). The LH was shown to be associated with variability in glucose level, hypoglycemia, and higher insulin consumption (6).

\section{Objectives}

The present study aimed to assess the prevalence of LH and to identify its association with a number of factors including systematic rotation of injection sites and needle length. In addition, the study assessed the association be- tween LH and glycemic control with the goal of reducing $\mathrm{LH}$ and its potential consequences on glycemic control.

\section{Patients and Methods}

\subsection{Study Population and Data Collection}

A clinical case-series study was conducted at the National Center for Diabetes, Endocrinology and Genetics (NCDEG) in Amman, Jordan. A total of 1090 insulintreated patients with T2DM were enrolled from October 2011 to January 2012. The presence of LH was determined by inspection and palpation of insulin injection sites and was classified into grades 0 through 3: grade 0 , no changes; grade 1 , visible hypertrophy of fat tissue but palpably normal consistency; grade 2, massive thickening of fat tissue with higher consistency; and grade 3, lipoatrophy.

At enrollment, the researcher interviewed the patients, obtained anthropometric and blood pressure measurements, and collected relevant clinical data. Laboratory

Copyright (C) 2015, Research Institute For Endocrine Sciences and Iran Endocrine Society. This is an open-access article distributed under the terms of the Creative Commons Attribution-NonCommercial 4.0 International License (http://creativecommons.org/licenses/by-nc/4.0/) which permits copy and redistribute the material just in noncommercial usages, provided the original work is properly cited. 
data were obtained from the medical records. These data were as follows:

(A) Socio-demographic characteristics such as age, sex, duration of T2DM, educational level, and duration of insulin treatment; (B) Anthropometric measurements such as height, weight, waist circumference (WC), and blood pressure; (C)Clinical characteristics such as types of insulin, number of insulin injections per day, sites of insulin injection, and needle size; (D) Laboratory measurements including glycosylated hemoglobin (HbA1c) and lipid profile.

\subsection{Variables' Definitions}

The WC was measured by a non-stretchable tailors tape at a point midway between the iliac crest and the costal margin at the horizontal plane, and was considered high if it exceeded $91.6 \mathrm{~cm}$ in men and $88.5 \mathrm{~cm}$ in women (7). Blood pressure was measured in a seated position after five minutes of rest by using standardized sphygmomanometer EN 1060 with a cuff circumference of 24 to $32 \mathrm{~cm}$ to cover $80 \%$ of the upper arm with the arm at heart level. Patients were considered hypertensive or dyslipidemic if they were already on antihypertensive or dyslipidemic medications, or if they met the American Diabetes Association (ADA) criteria of hypertension and dyslipidemia (8). Weight and height were measured with the subjects wearing light clothing and no shoes using Detecto scale and standiometer. Waist to height ratio was calculated as the waist circumference divided by the height in centimeters, and classified as normal if the value was $<0.5$ for $\leq 50$ years of age and $<0.6$ for $>$ 50 years of age. Glycemic control was classified, according to ADA criteria, as controlled if HbA1c was $<7 \%$, and uncontrolled if $\geq 7 \%$ (7).

\subsection{Ethical Consideration}

The study protocol was approved by the Ethics Committee of the NCDEG. The data were used only for scientific inquiry. Informed consent was obtained from all participants. Privacy of participants was respected and confidentiality was strictly maintained. The study carried no foreseen harm to participants.

\subsection{Statistical Analysis}

Statistical analysis was performed using the SPSS (SPSS Inc, Chicago, Illinois, the United States). Initially, the data were checked for data entry errors, which were corrected by referring to original data sources. Descriptive statistics were obtained, such as mean and median values for continuous variables and proportions for categorical variable. The prevalence of LH was obtained. Associations between LH and selected factors were assessed for statistical significance using the Chi square test. Multivariate logistic regression was used for the adjustment of potential confounders. P values $\leq 0.05$ was considered statistically significant.

\section{Results}

\subsection{Participants' Characteristics}

This study included 1090 insulin-treated patients with T2DM ( 515 males and 575 females) aged between 20 to 89 years with a mean (SD) age of 57.1 (10.3) years (range, 2089 years). The median T2DM duration was 13.5 years (interquartile range [IQR], $9-20$ years). Over $43 \%$ of patients had more than high school education.

The mean (SD) insulin treatment duration was 4.6 (5.0) years, and the median was 3 years (IQR, 1.5-6.0 years) indicating skewness to the left. Overall, $87.4 \%$ of patients were injecting insulin in the abdomen and $65 \%$ were injecting insulin three times or more per day and $89.9 \%$ were using injection needle of 8-mm length.

The prevalence of obesity was $67.9 \%, 93.7 \%$ of patients had high WC, 92.4\% had hypertension, 61\% changed insulin injection sites frequently, and $58.3 \%$ were on treatment by Mixtard insulin. Regarding laboratory results, $9.8 \%$ of patients had controlled glucose levels (HbA1c < $7 \%$ ) and $96.9 \%$ of patients had dyslipidemia.

\subsection{Prevalence of Lipohypertrophy in Insulin-Treat- ed Patients With Type 2 Diabetes Mellitus}

Table 1 shows the prevalence of $\mathrm{LH}$ by selected variables and the significance of observed differences. The overall prevalence of LH in the study population was $37.3 \%$. Females were significantly more likely to have LH than males were $(\mathrm{P}=0.005)$. Prevalence of $\mathrm{LH}$ was significantly higher among illiterate patients $(50.4 \%$; $<0.001)$ and those with body mass index between 35 and $39.9 \mathrm{~kg} / \mathrm{m}^{2}$ (45.8\%; $\mathrm{P}=0.034$ ). In addition, $50 \%$ of patients with diabetes duration $\geq 20$ years had $\mathrm{LH}$ and $53.5 \%$ of patients with duration of insulin treatment $\geq 10$ years had LH. The prevalence of $\mathrm{LH}$ was $41 \%$ in patients with HbA1c $\geq$ $9 \%$ and $41 \%$ in patients on Mixtard insulin. Patients using needle size of $12 \mathrm{~mm}$ were more likely to have LH than patients using needle size of $8 \mathrm{~mm}$. Prevalence of LH was also higher in those who did not rotate injection sites (84.6\%) than in those who rotated the sites $(\mathrm{P}<0.001)$.

Stepwise multivariate logistic regression analysis was used to identify factors independently related to LH. Results are shown in Table 2 in which each variable was adjusted for all other variables in the table. All variables that were statistically significant in the bivariate analysis (Table 1) but lost their significance were removed from the model by the stepwise procedure. Results showed that patients who did not rotate their insulin-injection sites were 118 times more likely to have LH compared to those who systematically change their injection sites. Patients with HbA1c levels $\geq$ $7 \%$ were at several fold increased risk of developing LH than those having HbA1c $<7 \%$ (Table 2). Patients with duration of insulin treatment $\geq 10$ years were 46.4 times more likely to develop LH than those with insulin treatment duration of $<1$ year were $(\mathrm{P}<0.001)$. In addition, those using insulin 
injection needle size of $12 \mathrm{~mm}$ were 7.4 times more likely to develop LH than those using injection needle of 8-mm length were $(\mathrm{P}<0.001)$. Patients with duration of T2DM $\geq 20$ years were 3.8 times more likely to develop LH than those with duration of $<1$ year were $(\mathrm{P}=0.044)$.
In summary, lack of systemic rotation of injection sites was the strongest predictive factor for developing $\mathrm{LH}$, followed by duration of insulin therapy, duration of diabetes, and needle size. Lack of glycemic control was also strongly associated with LH as compared to ideal glycemic control.

Table 1. Prevalence of Lipohypertrophy by Selected Variables and the Significance of Observed Differences

\begin{tabular}{|c|c|c|c|}
\hline Variable & Lipohypertrophy, No. (\%) & No Lipohypertrophy, No. (\%) & PValue \\
\hline Age, $y$ & & & 0.005 \\
\hline$<50$ & $53(29.0)$ & $130(71.0)$ & \\
\hline $50-59$ & $121(35.1)$ & $224(64.9)$ & \\
\hline $60-69$ & $157(39.9)$ & $236(60.1)$ & \\
\hline$\geq 70$ & $78(46.2)$ & $118(53.8)$ & \\
\hline Sex & & & $<0.001$ \\
\hline Male & $131(25.4)$ & $384(74.6)$ & \\
\hline Female & $384(48.3)$ & $191(51.7)$ & \\
\hline Duration of Diabetes, $y$ & & & $<0.001$ \\
\hline $1-4$ & $20(21.1)$ & $75(78.9)$ & \\
\hline $5-9$ & $59(31.6)$ & $128(68.4)$ & \\
\hline $10-14$ & $99(33.0)$ & $201(67.0)$ & \\
\hline $15-19$ & $92(40.0)$ & $138(60.0)$ & \\
\hline$\geq 20$ & $139(50.0)$ & $139(50.0)$ & \\
\hline Insulin Treatment Duration, y & & & $<0.001$ \\
\hline$<1$ & $58(63.0)$ & $34(37.0)$ & \\
\hline $1-2.4$ & $53(13.7)$ & $333(86.3)$ & \\
\hline $2.5-4.9$ & $105(43.0)$ & $139(57.0)$ & \\
\hline 5-9.9 & $102(51.5)$ & $96(48.5)$ & \\
\hline$\geq 10$ & $91(53.5)$ & $79(46.5)$ & \\
\hline Education Level & & & $<0.001$ \\
\hline Illiterate & $68(50.4)$ & $67(49.6)$ & \\
\hline$\leq$ high school & $190(39.6)$ & $290(60.4)$ & \\
\hline$>$ high school & $151(31.8)$ & $324(68.2)$ & \\
\hline Body Mass Index, $\mathrm{kg} / \mathrm{m}^{2}$ & & & 0.034 \\
\hline$<25$ & $28(35.0)$ & $52(65.0)$ & \\
\hline 25-29.9 & $100(37.5)$ & $167(62.5)$ & \\
\hline 30-34.9 & $118(31.7)$ & $254(68.3)$ & \\
\hline 35-39.9 & $110(45.8)$ & $130(54.2)$ & \\
\hline$\geq 40$ & $51(39.5)$ & $78(60.5)$ & \\
\hline Glycosylated Hemoglobin (HbA1c), \% & & & $<0.001$ \\
\hline$<7$ & $19(17.8)$ & $88(82.2)$ & \\
\hline $7-7.9$ & $95(35.4)$ & $173(64.6)$ & \\
\hline 8-8.9 & $135(41.4)$ & $191(58.6)$ & \\
\hline$\geq 9$ & $159(41.0)$ & $229(59.0)$ & \\
\hline Systematic Rotation of Insulin Injection Site & & & $<0.001$ \\
\hline Yes & $59(8.7)$ & $616(91.3)$ & \\
\hline No & $351(84.6)$ & $64(15.4)$ & \\
\hline Needle Size, mm & & & $<0.001$ \\
\hline 8 & $336(34.3)$ & $643(65.7)$ & \\
\hline 12 & $73(66.4)$ & $37(33.6)$ & \\
\hline Frequency of Insulin Injection, No. & & & 0.002 \\
\hline 1 & $90(28.2)$ & $229(71.8)$ & \\
\hline 2 & $27(43.5)$ & $35(56.5)$ & \\
\hline$\geq 3$ & $292(40.6)$ & $427(59.4)$ & \\
\hline
\end{tabular}


Al Ajlouni Met al.

\begin{tabular}{|c|c|c|}
\hline Variable & Adjusted Odds Ratio & PValue \\
\hline \multicolumn{3}{|c|}{ Glycosylated Hemoglobin (HbA1c), \% } \\
\hline$<7$ & 1 & \\
\hline $7-7.9$ & 7.15 & 0.001 \\
\hline $8-8.9$ & 5.66 & 0.003 \\
\hline$\geq 9$ & 7.11 & 0.001 \\
\hline \multicolumn{3}{|c|}{ Duration of Diabetes, $y$} \\
\hline $1-4$ & 1 & \\
\hline $5-9$ & 1.67 & 0.816 \\
\hline $10-14$ & 0.79 & 0.724 \\
\hline $15-19$ & 1.62 & 0.463 \\
\hline$\geq 20$ & 3.77 & 0.044 \\
\hline \multicolumn{3}{|c|}{ Duration of Insulin Treatment, y } \\
\hline$<1$ & 1 & \\
\hline $1-2.4$ & 4.07 & 0.017 \\
\hline 2.4-4.9 & 7.91 & $<0.001$ \\
\hline 5-9.5 & 34.68 & $<0.001$ \\
\hline$\geq 10$ & 46.42 & $<0.001$ \\
\hline \multicolumn{3}{|c|}{ Needle Size, mm } \\
\hline 8 & 1 & \\
\hline 12 & 7.41 & $<0.001$ \\
\hline \multicolumn{3}{|c|}{ Systematic Rotation of Insulin Injection Site } \\
\hline Yes & 1 & \\
\hline No & 118.19 & $<0.001$ \\
\hline
\end{tabular}

\section{Discussion}

The prevalence rates of $\mathrm{LH}$ in insulin-treated patients with T2DM have been variable in different studies. In the present study, LH was observed in $37.3 \%$ of insulintreated patients with T2DM (grade 1, 27.4\%; grade 2, 9.7\%; and grade $3,0.2 \%)$, a prevalence much higher than the rate reported by Hauner et al. (5) and Kasha et al (9), where the prevalence of $\mathrm{LH}$ was reported to be $3.6 \%$ and $14.5 \%$, respectively. However, a much higher rate (64.4\%) was reported by a more recent study (6). The variability in prevalence of LH in studies may be related to lack of routine examination of most of insulin-treated patients with T2DM for the presence of LH in diabetic clinics. In the present study, all patients were thoroughly examined for LH to avoid possible underestimation of routinely generated data. It may also be related to the case-mix of patients regarding duration of insulin therapy and the standard of care they received, ie, the systematic rotation of insulin-injection sites and the used needle sizes. The NCDEG, where the study was conducted, is a referral center that admits patients from all over the country. However, patients may also seek treatment in the Center directly. Our patient-mix, therefore, is likely to over-rep- resent advanced patients with longer duration of their disease who are more likely to have LH.

Changing insulin injection sites has been shown to be associated with better glycemic control. Chowdhury et al. (10) reported that HbA1c fell from $10.9 \%$ to $7.8 \%$ after changing insulin injection sites, leading to reduction of the dose of insulin by two to four units over the following three months. This effect was also reported by other studies $(10,11)$

The LH has been reported to be inversely correlated with the frequency of rotating insulin injection sites in many studies (5, 9-14). These findings are consistent with our results where LH was associated with rotating injection sites and better glycemic control. In our study, illiterates were more likely to have LH than educated patients were, suggesting a need for closer supervision and more effort in explaining this complication and its prevention to illiterate patients. Our study showed a significant positive association between duration of insulin treatment and LH. Duration of insulin treatment was identified as an independent risk factor for LH in many studies $(9,11)$.

Consistent with a number of previous studies con- 
ducted by Kasha et al. and Malwa et al. $(9,13)$, the present study showed that LH was significantly associated with the length of insulin injection needle. The LH is a common problem in insulin-treated Jordanian patients with T2DM. More efforts are needed to educate patients and health workers on it. Interventions such as using of shorter needle lengths and frequent rotation of the insulin injection sites are essential interventions to treat or prevent LH.

\section{Authors' Contributions}

Mousa Abujbara wrote, supervised, and edited the manuscript. Mo'men Al Ajlouni was primarily responsible for the conception and design of the study in addition to data collection. Anwar Batieha supervised each step of the research and was the statistical advisor. Kamel Ajlouni was the guarantor of this work and reviewed the manuscript.

\section{References}

1. Intensive blood-glucose control with sulphonylureas or insulin compared with conventional treatment and risk of complications in patients with type 2 diabetes (UKPDS 33). UK Prospective Diabetes Study (UKPDS) Group. Lancet. 1998;352(9131):837-53.

2. Fujikura J, Fujimoto M, Yasue S, Noguchi M, Masuzaki H, Hosoda $\mathrm{K}$, et al. Insulin-induced lipohypertrophy: report of a case with histopathology. Endocr J. 2005;52(5):623-8.

3. Seyoum B, Abdulkadir J. Systematic inspection of insulin injec- tion sites for local complications related to incorrect injection technique. Trop Doct.1996;26(4):159-61.

4. Richardson T, Kerr D. Skin-related complications of insulin therapy: epidemiology and emerging management strategies. Am J Clin Dermatol. 2003;4(10):661-7.

5. Hauner H, Stockamp B, Haastert B. Prevalence of lipohypertrophy in insulin-treated diabetic patients and predisposing factors. Exp Clin Endocrinol Diabetes. 1996;104(2):106-10.

6. Blanco M, Hernandez MT, Strauss KW, Amaya M. Prevalence and risk factors of lipohypertrophy in insulin-injecting patients with diabetes. Diabetes Metab. 2013;39(5):445-53.

7. Carr DB, Utzschneider KM, Hull r. l. , Kodama K, Retzlaff BM, Brunzell JD, et al. Intra-abdominal fat is a major determinant of the National Cholesterol Education Program Adult Treatment Panel III criteria for the metabolic syndrome. Diabetes Metab. 1996;53:2087-94.

8. American Diabetes A. Standards of medical care in diabetes--2011. Diabetes Care. 2011;34 Suppl 1:S11-61.

9. Kasha Z, Haiheydan Z, Akha O, Akbarzadeh S. Prevalence of lipodystrophy associated with recombinant insulin. Mazandaran Uni Med Sci J. 2008;18:9.

10. Chowdhury TA, Escudier V. Poor glycaemic control caused by insulin induced lipohypertrophy. BMJ. 2003;327(7411):383-4.

11. Hambridge $K$. The management of lipohypertrophy in diabetes care. Br J Nurs. 2007;16(9):520-4.

12. Sea-de Ibarra L, Gallego F. Factors related to Lipohypertrophy in insulin-treated diabetic patients: role of educational intervention. Practical Diab Int.1998;15(1):9-11.

13. Malwa G, Balami D, Deshmukh S, Groft M, Bodmer C, Patel M. Insulin-induced Lipohypertrophy, past present and future: are we lose the bottle. Endocrine. 2010;22:268.

14. Vardar B, Kizilci S. Incidence of lipohypertrophy in diabetic patients and a study of influencing factors. Diabetes Res Clin Pract. 2007;77(2):231-6. 